\title{
Incidencia de factores institucionales y de la autoestima en las trayectorias académicas de estudiantes con discapacidad
}

Ana-Inés Mainardi-Remis

\section{RESUMEN}

La educación se encuentra frente al desafio de tener que brindar respuestas concretas al reclamo por acciones inclusivas que aborden la diversidad y eliminen las barreras que excluyen y etiquetan las diferencias. Este artículo refleja uno de los hallazgos obtenido en una investigación que incursionó en el conocimiento de factores institucionales y psicosociales (en particular la autoestima) que impactan en el trayecto y rendimiento académico de estudiantes con discapacidad; el estudio se realizó en dos universidades argentinas. Los resultados encontrados pretenden propiciar la reflexión y construcción de ámbitos de intervención que promuevan la participación activa, la revalorización de los derechos humanos y la mejora de nuestros servicios educativos en vista a la real inclusión de las personas con discapacidad.

Palabras clave: autoestima, discapacidad, inclusión, trayectorias académicas, Argentina.

\footnotetext{
Ana-Inés Mainardi-Remis

anamainardiremis@gmail.com Argentina. Doctora en Educación, Facultad de Filosofía y Letras, Universidad Nacional de Cuyo, Argentina. Becaria posdoctoral del Consejo Nacional de Investigaciones Científicas y Técnicas (CONICET), Argentina; miembro del Instituto Nacional de Investigaciones en Psicología y Educación (INIPE); docente en la Facultad de Humanidades, Universidad Nacional de Salta, Argentina. Temas de Investigación: universidad, diversidad e inclusión; trayectorias académicas, representaciones y perfiles psicosociales en estudiantes con discapacidad.
} 


\section{Incidência de fatores institucionais e da autoestima nas trajetórias académicas de estudantes com deficiência}

\section{RESUMO}

A educação se encontra frente ao desafio de ter que brindar respostas concretas ao reclamo por ações inclusivas que abordem a diversidade e eliminem as barreiras que excluem e etiquetam as diferencias. Este artigo reflete um dos descobrimentos obtido numa pesquisa que se inseriu no conhecimento de fatores institucionais e psicossociais (em particular a autoestima) que impactam no trajeto e rendimento académico de estudantes com deficiência; o estudo se realizou em duas universidades argentinas. Os resultados encontrados pretendem propiciar a reflexão e a construção de âmbitos de intervenção que promovam a participação ativa, a reavaliação dos direitos humanos e a melhora de nossos serviços educativos tendo em vista à real inclusão das pessoas com deficiência.

Palavras chave: autoestima, deficiência, inclusão, trajetórias académicas, Argentina.

\section{Incidence of institutional factors and self-confidence on the academic careers of students with disabilities}

\section{ABSTRACT}

Education faces the challenge of having to provide concrete responses to the call for inclusive actions able to address diversity and remove the barriers that exclude and label differences. This article discloses one of the findings of a research study that explored the knowledge of institutional and psychosocial factors (in particular self-confidence) that impact the academic career and performance of disabled students. The study was carried out at two Argentine universities. The results intend to encourage a new way of thinking about the problem and the construction of intervention areas that promote active participation, the revaluation of human rights and the improvement of the country's educational services with a view to the real inclusion of people with disabilities.

Key words: self-confidence, disability, inclusion, academic careers, Argentina.

Recepción: 13/03/17. Aprobación: 14/09/17. 


\section{Introducción}

El último cuarto de siglo nos coloca frente a paradigmas que consideran la revalorización positiva de las diferencias, la atención en y para la diversidad y la educación inclusiva como conceptos y herramientas apropiadas en la formación de las personas con discapacidad (PCD).

La universidad, como una de las organizaciones referentes de la sociedad, no escapa a estas demandas por lo que no debe restringir su función sólo a generar conocimientos y desarrollar habilidades sino que su rol es también el de enseñar, fomentar y desarrollar valores y actitudes, y poder garantizar en su seno un elevado nivel de protección a sus miembros frente a los riesgos derivados de sus actividades (Orell y Sainz, 2006).

En este marco, diversos autores coinciden en manifestar la relevancia que adquiere el estudio de aquellos factores que pudieran desarrollarse como facilitadores $\mathrm{u}$ obstaculizadores en el trayecto formativo de los estudiantes (Kaplan et al., 2001; González-Lizárraga, 2011; Garrido, 2012). Conocer y comprender la presencia e incidencia de algunos de estos aspectos en el ámbito de la educación superior puede contribuir a generar y fortalecer las condiciones de acceso, permanencia y posterior egreso de los estudiantes en general y de aquellos con discapacidad en particular, promoviendo espacios de real inclusión.

Este escrito presenta un recorte que expone los principales datos obtenidos en un estudio que incursionó mediante el empleo de metodología cuantitativa y cualitativa, en los perfiles psicosociales y el impacto de diversos factores institucionales en las trayectorias académicas de estudiantes con discapacidad que desarrollan sus itinerarios formativos en la Universidad Nacional de Salta (UNSa) y en la Universidad Nacional de Cuyo (UNCuyo). Si bien ambas instituciones argentinas de gestión estatal cuentan con programas específicos para la inclusión de PCD, es posible evidenciar que muchos objetivos no han sido conseguidos plenamente, resultando necesarias líneas de investigación que permitan el análisis de la temática a fin de formular nuevas estrategias que promuevan la equiparación de oportunidades, fortalezcan el desarrollo integral de cada persona y aborden la diversidad en orden a la inclusión.

A continuación efectuaremos un recorrido desde los principales sustentos conceptuales que subyacen a este estudio, para luego adentrarnos en los resultados alcanzados en relación con dos aspectos: a) la vinculación de la autoestima personal con el rendimiento académico de los alumnos con discapacidad y, b) la presencia de algunos aspectos favorecedores o limitadores que intervienen en las trayectorias académicas de personas con discapacidad.

\section{Encuadre teórico}

En contextos caracterizados por gran heterogeneidad, una oferta educativa homogénea se traduce en trayectorias y resultados desiguales. Por eso, durante mucho tiempo, la educación se ha inclinado más a profundizar las desigualdades como consecuencia de no tener en cuenta las diferencias de los estudiantes. En la actualidad y "basados en la defensa y apoyo que se ha dado al respeto de la diversidad humana, se plantea la inclusión de las minorías a todos aquellos escenarios de donde fueron excluidos" (Soto-Builes et al., 2008: 20).

En el tránsito universitario, los estudiantes pasan por una serie de desafíos donde aprenden progresivamente a ejercer este rol (ser estudiante). A medida que pasa el tiempo en el transcurso universitario, los proyectos profesionales y personales se van definiendo y las vocaciones se revelan, será entonces - en este caminar - que los estudiantes van integrándose y adquiriendo ciertas capacidades que los hacen "sobrevivir" a este proceso (Gonzalez-Lizárraga, 2011).

Esta perspectiva invita al análisis de los estudiantes como sujetos concretos que se encuentran insertos en las instituciones, que cuentan con trayectorias, experiencias y voz propias. 
"La trayectoria educativa no es un proceso lineal y caracterizado desde sus comienzos, sino que más bien, es un entramado que se constituye en constante comunicación" (Garrido, 2012: 7) con el contexto educacional y con la dimensión social en general. En este sentido, la heterogeneidad y diversidad de componentes que integran una determinada cultura y que se subjetivan de manera especial en cada individuo, imprimen la particularidad de cada trayectoria educativa (Garrido, 2012).

La temática de los recorridos universitarios ha tomado relevancia en los últimos años para la investigación educativa (Kaplan et al., 2001; GonzalezLizárraga, 2011; Garrido, 2012) en la medida en que es posible conocer sus procesos, los cuales implican distintas prácticas desarrolladas por los estudiantes.

En este sentido, son múltiples las trayectorias de los sujetos mediante la experiencia estudiantil y "están dominadas por el encuentro de varios obstáculos que tienen que ver con el tipo de organización universitaria, el contenido y la finalidad de los propios estudios" (González-Lizárraga, 2011: 91).

En líneas generales entonces, se entiende por trayectoria académica (referida al ámbito universitario) al recorrido o parte del recorrido que realizan los estudiantes bajo condiciones y características diferentes de ingreso y que será delimitada de diversas formas por la organización universitaria, las vinculaciones, las carreras profesionales que el estudiante elige, así como su contexto social y experiencial de referencia (González-Lizárraga, 2011).

Este nivel de involucramiento de los estudiantes en la vida académica propicia la introducción al estudio de lo que se conoce como rendimiento académico en términos del promedio obtenido a lo largo de la trayectoria (González-Lizárraga, 2011). Tal como se expresa en la literatura, el rendimiento académico puede ser entendido como la medida de los conocimientos y capacidades del estudiante que indica lo que éste ha aprendido a lo largo del proceso formativo, suponiendo también la capacidad del alumno para responder a los estímulos educativos (Castro et al., 2001; Aparicio, 2009; Serrano-Barquín et al., 2013).

Aparicio (2009) efectúa una distinción de tres grupos de factores que impactan en el rendimiento: factores socioculturales, escolares y personales. Entre los socioculturales, se encuentran los procesos de socialización dentro de la familia y la institución educativa, el origen y marco social de referencia del sujeto y la cultura familiar.

Entre los factores escolares, está la realidad institucional educativa, el rol de la calidad de la enseñanza, las relaciones profesor-alumno y el clima en el proceso de enseñanza y aprendizaje como los más representativos (Aparicio, 2009).

Finalmente, están los factores personales; en este caso el autoconcepto y autoestima son dos de los aspectos más analizados en relación con el rendimiento: "Estudios realizados en distintos contextos (España, EUA, África, Canadá) muestran la alta incidencia sobre el mismo (cf. Reyes Tejada, 2003)" (Aparicio, 2009: 27).

En este artículo se pretende profundizar en la noción de autoestima, reconocida como un indicador del desarrollo personal fundado en la valoración positiva, negativa o neutra que cada persona hace de sus características cognitivas, físicas y psicológicas (Toro-Vargas, 1994; Huanco-Flores, 2004; Polo et al., 2012). Tal valoración se construye sobre la base de la opinión que cada persona tiene sobre sí misma, a partir de los atributos que le otorgan las características mencionadas.

Según como se encuentre la autoestima, ésta tendrá influencia en los estados anímicos y la forma de relacionarse con el entorno. De esta manera, una autoestima adecuada, vinculada a un concepto positivo de sí mismo, potenciará la capacidad de las personas para desarrollar sus habilidades y aumentará el nivel de seguridad personal, mientras que una autoestima baja enfocará a la persona hacia la inseguridad (Toro-Vargas, 1994).

Si bien no resulta fácil encontrar bibliografía que 
analice esta variable en estudiantes con discapacidad en particular, es factible identificar en diferentes literaturas la creencia de que la persona con discapacidad carece de la posibilidad de percibirse a sí misma y de manifestar sentimientos de confianza y valoración positivas. Ocurre, en muchas ocasiones, que el resto de los individuos tienden a conversar sobre ellas en sus presencias (aunque invisibilizadas), con términos desvalorizadores sobre lo que pueden realizar o lo que son (Castilla, 2006).

[...] el yo de las personas con discapacidad crece y se desarrolla del mismo modo que el de las personas sin discapacidad, lo que sucede es que, con frecuencia, recibe influencias negativas, enfrentándose desde la infancia, en muchos casos, a rechazo social y a experiencias negativas en las relaciones interpersonales, que la desvalorizan y frustran. Estas circunstancias conducen a la hipótesis de que las personas con discapacidad son un grupo en riesgo de desarrollar un autoconcepto negativo. [...] De hecho, son muchos los autores que establecen una estrecha relación entre el concepto que sobre sí mismas tienen las personas discapacitadas y sus opciones para integrarse en los diferentes ámbitos en los que normalmente se desenvuelven (Polo et al., 2012: 88, 89).

Tomando en consideración los conceptos hasta aquí enunciados, puede vislumbrarse que los factores asociados a las trayectorias académicas son múltiples y es necesario advertir la incidencia que, sobre el rendimiento, pueden tener muchos de éstos (Aparicio, 2009).

\section{Diseño metodológico}

Dado el complejo entramado de los aspectos y factores analizados, se desarrolló un diseño metodológico que facilite el tratamiento integral de los mismos. Por lo tanto, el estudio se enmarcó en un abordaje mixto (Hernández-Sampieri et al., 2010) combinando componentes cuantitativos y cualitativos en la investigación, en orden a conocer los perfiles de autoestima de los estudiantes universitarios con discapacidad, así como analizar la incidencia de algunos factores en el recorrido y rendimiento académico de los mismos. Los datos cuantitativos y cualitativos se recogieron y analizaron más o menos al mismo tiempo, de modo que se empleó un diseño concurrente.

\section{Muestra y sujetos de la investigación}

Se utilizó un muestreo no probabilístico intencional o de conveniencia (Baranger, 2009), a partir del cual se trabajó con los participantes que se encontraban dispuestos y disponibles para ser estudiados.

La muestra quedó conformada por 40 estudiantes de distintas carreras de grado de la Universidad Nacional de Guyo ( $\mathrm{n}=21)$ y de la Universidad Nacional de Salta $(\mathrm{n}=19)$.

Respetando los criterios de "autodeclaración" ("yo me declaro persona con discapacidad") y "autoreconocimiento" "“yo me reconozco como persona con discapacidad"), se les solicitó el consentimiento informado a cada uno de los estudiantes. A este respecto, vale la salvedad de que existen sujetos que declaran que tienen una discapacidad, referenciando la deficiencia (disfunción o ausencia orgánica), sin embargo no se reconocen como persona con discapacidad. Y en otros casos, algunos individuos se reconocen como persona con discapacidad, pero no lo declaran, por distintos motivos de índole personal, la mayoría asociados al temor de ser rechazados, discriminados y/o excluidos.

\section{Procedimiento}

Como señalamos anteriormente, se trabajó en dos instituciones universitarias de gestión pública. La elección de las mismas obedeció, por un lado, al reconocimiento del funcionamiento de distintos programas que desarrolla cada universidad para la inclusión de sus estudiantes con discapacidad, lo 
cual permitió la aproximación a aspectos posibles de comparar y, por otro lado, a las posibilidades de la investigadora de acceder y permanecer en éstas para abordar la problemática y trabajar en terreno durante el tiempo que lo requiriera.

La recolección de los datos se llevó a cabo en diferentes momentos y espacios, siguiendo la disponibilidad de cada estudiante, por lo que fue de forma personalizada, individual y a modo de entrevista. En todos los casos, a fin de crear un clima confiable y respetuoso, las instancias de encuentro se desenvolvieron en situación de ambiente natural (contexto universitario).

Todos los estudiantes que participaron lo hicieron de manera voluntaria y previa notificación de consentimiento informado. Se respetó el secreto estadístico de cada institución, conservando el anonimato de los sujetos.

Si bien el instrumento fue proporcionado en formato papel, no obstante, teniendo en cuenta el tipo de discapacidad de algunos estudiantes, el mismo se aplicó de forma oral siendo la investigadora quien registrara las respuestas brindadas por el participante. Tales instancias, bajo consentimiento del sujeto, fueron grabadas para extraer con mayor riqueza algunas expresiones de los estudiantes. Dada la rigurosidad del instrumento y las particularidades de la población, los estudiantes precisaron de un tiempo que osciló entre 60 y 180. Se solicitó que en la medida de lo posible, respondieran el total de los cuestionamientos y expresaran simplemente lo que pensaban, dejando en claro que su colaboración no afectaría ni a favor ni en contra su desarrollo en la vida académica.

\section{Instrumentos y análisis de datos}

La recolección de datos se efectuó a partir de la aplicación de un cuestionario semiestructurado, elaborado específicamente para este estudio, el cual quedó compuesto por diferentes técnicas cuantitativas y herramientas cualitativas que permitieron la posterior descripción y aproximación a los factores psicosociales (autoestima) y contextuales y su relación con la trayectoria académica (rendimiento) de los estudiantes.

\section{Análisis cuantitativos}

A nivel cuantitativo se trabajó con preguntas cerradas, que posibilitaron conocer algunas variables sociodemográficas, organizacionales- institucionales, situación académica de los estudiantes (rendimiento) y además, se aplicaron pruebas psicométricas, entre las que destaca el test Autoestima: Autoexamen de Cirilo Toro-Vargas (1994). Los datos fueron analizados mediante el programa Statistical Package for Social Sciences (SPSS) versión 22 (2013).

Para el desarrollo del estudio (dadas las características de la muestra y su abordaje), se emplearon pruebas no paramétricas. No obstante, pudo aplicarse la $t$ de Student que, aunque es una medida paramétrica, tiene la ventaja de que permite el tratamiento de muestras pequeñas $(\mathrm{N}=30)$ aportando datos valiosos sobre la capacidad de discriminación de cada ítem, puesto que compara las medias y permite visualizar si las diferencias encontradas son significativas.

Asimismo, con el fin de indagar cómo se agrupan en cada estudiante las variables, se llevó a cabo un análisis de conglomerados, también conocido como análisis de clúster (Baranger, 2009; Hernández-Sampieri et al., 2010). Este análisis brinda la información sobre en qué conglomerado se ubica a cada sujeto, es decir cómo se configuran los perfiles de cada estudiante en función de las variables abordadas (autoestima y rendimiento académico).

Puesto que la muestra con la que se trabajó es pequeña, resultó imposible emplear la técnica del análisis factorial debido a las condiciones que éste requiere, por lo que para aproximarnos a la validación, se recurrió a los aportes del análisis del índice de homogeneidad de las escalas utilizando el coeficiente alpha de Cronbach. De esta manera, con el 
objetivo de analizar en qué medida cada enunciado de la escala evalúa el mismo concepto de forma equivalente, se calcularon los valores de alpha para las dimensiones del test de autoestima a partir de los cuales se puede corroborar su consistencia interna.

A este respecto, para la categorización de los grados de autoestima se consideró el instrumento completo según la tabla que propone el autor Cirilo Toro-Vargas (1994) (tabla 1).

- Autoestima alta negativa (-): refiere a la sobreestima, lo cual se traduce en actitudes individualistas y profundamente egoístas.

- Autoestima alta positiva (+): se manifiesta en el desarrollo de una personalidad más plena y una percepción más satisfactoria de la vida.

- Autoestima baja (+): la persona presenta ciertos grados de inseguridad sobre sí misma, pero eso no le impide fijarse metas y aspiraciones propias, superar dificultades y tener una percepción medianamente satisfactoria de la vida.

- Autoestima baja (-): falta confianza en uno mismo para abordar, desde una perspectiva positiva, los retos que pudiera presentar la vida, provocando que la calidad de vida no sea totalmente óptima.

Asimismo, para realizar un análisis más completo, se tomaron los aportes de Huanco (2004), quien reconoce cinco dimensiones en la autoestima:

- Dimensión física: consciencia del valor físico.

- Dimensión cognoscitiva: confianza en poder descubrir cómo son, cómo funcionan y cómo se relacionan las cosas.

- Dimensión afectiva: capacidad de sentir, expresar los sentimientos, sensaciones, temores y de establecer vínculos afectivos.

- Dimensión socio-afectiva: sentimiento de sentirse aceptado y de pertenencia a un grupo social.

- Dimensión ética positiva (+): seguridad acerca de lo que se considera bueno y de las conductas correctas que se deben realizar para las personas y para la sociedad. Dimensión ética negativa (-): conductas soberbias.

Tabla 1. Interpretación de la autoescala

\begin{tabular}{|c|c|}
\hline Intervalos de puntuación & Significado \\
\hline $40-73$ & Autoestima baja (negativa) \\
\hline $74-83$ & Autoestima baja (positiva) \\
\hline $84-103$ & Autoestima alta (positiva) \\
\hline $104-160$ & Autoestima alta (negativa) = sobreestima \\
\hline
\end{tabular}

Fuente: Test de Autoestima Toro-Vargas (1994). 
Los primeros análisis, siguiendo el procedimiento establecido por el autor del instrumento (ToroVargas, 1994), arrojaron índices no adecuados de homogeneidad - y, por ende, coeficientes alpha descendidos-; por ello, se elaboró una matriz de configuración que, por el tamaño de la muestra, sólo se empleó para identificar una agrupación que permitiera el análisis psicométrico.

Mediante este procedimiento, se conformaron las subescalas (en algunos casos con ítems reversos) ${ }^{1}$ y se calcularon los índices de homogeneidad, destacándose que la totalidad de los ítems resulta homogénea, esto es, los indicadores de cada escala se diferencian en el mismo sentido que la escala en su totalidad, lo que expresa que evalúan una variable unitaria.
Mediante el cálculo del alpha de Cronbach se halla que los índices oscilan entre 0.59 y 0.78 , coeficientes que se consideran de confiabilidad aceptable a buena. Por su parte, el instrumento completo (en 40 ítems) alcanzó un índice aceptable: 0.64.

Respecto de la potencialidad discriminativa de los ítems a través del contraste entre grupos opuestos (11 estudiantes en el superior y el mismo número en el inferior) mediante el estadístico $t$ de Student, los resul$\operatorname{tados}^{2}$ manifiestan que todos los indicadores resultan discriminativos. En suma, la escala de autoestima queda conformada como se observa en la tabla 2.

Finalmente, para medir la variable rendimiento académico se utilizaron los promedios de los estudiantes al momento del relevamiento, según lo establecido

Tabla 2. Conformación de la escala de Autoestima a partir de la muestra $(N=40)$

\begin{tabular}{|l|c|}
\hline \multicolumn{1}{|c|}{ Escala } & \multicolumn{1}{|c|}{$\boldsymbol{N}^{\circ}$ de ítems } \\
\hline Dimensión afectiva positiva (+) & 10 \\
\hline Dimensión socio-afectiva negativa (-) & 8 \\
\hline Dimensión ética negativa (-) (soberbia) & 4 \\
\hline Dimensión ética positiva (+) & 5 \\
\hline Dimensión cognoscitiva positiva (+) & 4 \\
\hline Dimensión física negativa (-) & 37 \\
\hline Total de ítems & \\
\hline
\end{tabular}

Fuente: elaboración propia, a partir del análisis efectuado con el SPSS.

\footnotetext{
${ }^{1}$ Por otra parte, se eliminaron tres ítems que no correlacionaban en dimensión alguna: $\mathrm{N}^{\circ}$ 5: Me siento triste, $\mathrm{N}^{\circ}$ 38: Me aterran los cambios, $\mathrm{N}^{\circ}$ 40: Me alegro cuando otros alcanzan el éxito en sus intentos.

${ }^{2}$ En todos los casos, $\mathrm{gl}=20$.
} 
en sus respectivos estados curriculares consultados en los correspondientes departamentos de alumnos. Tales puntajes han sido elegidos como instrumentos para la medición del rendimiento académico, ya que se trata de una medida que sintetiza el desempeño del estudiante a lo largo de su trayecto formativo no concluido (desde el ingreso a la carrera hasta el momento de la encuesta).

\section{Análisis cualitativos}

En la instancia cualitativa, se trabajó con análisis lexicométrico y asociación libre de palabras. Ambas herramientas permitieron trabajar a partir de la propia voz de los participantes, mediante la construcción de categorías explicativas que contribuyeron a profundizar en las principales representaciones asociadas a las limitaciones y fortalezas en las trayectorias de los estudiantes con discapacidad, a partir de una adaptación de las técnicas de Abric (2001).

La construcción de categorías explicativas permite la reducción de los datos, proceso que supone una simplificación, resumen y selección de la información para poder abordarla. El procedimiento consistió en encontrar y dar nombre a los patrones generales de respuesta (respuestas similares o comunes) respetando el sentido auténtico de los datos.

En este sentido, el empleo del Análisis lexicométrico permitió trabajar con las expresiones literales de los sujetos, vertidas en las respuestas a preguntas abiertas, a efectos de identificar regularidades y establecer a posteriori categorías que marquen las prioridades de los grupos respecto de sus apreciaciones sobre algunos aspectos organizacionales/institucionales.

Por su parte, el recurso metodológico de Asociación libre de palabras posibilitó a los sujetos de la investigación, establecer la asociación espontánea de palabras o nociones en torno a ciertos focos inductores considerados relevantes en el estudio, tales como: "aportes de los estudios universitarios", "facilidades y obstáculos en el acceso y permanencia en una universidad" y "modificaciones en el trayecto formativo", entre los más representativos. Se construyeron categorías explicativas a posteriori y se trabajaron según frecuencia de aparición.

\section{Resultados}

Para ofrecer mayor claridad en los resultados del análisis, primero se describirán los datos correspondientes a cada variable y posteriormente lo que se encontró como producto de la integración de las diferentes variables que se abordan en el estudio.

\section{Características generales de la muestra y situación académica}

La muestra total con la que se trabajó estuvo constituida por un $48 \%$ de estudiantes de sexo femenino y un $52 \%$ de estudiantes de sexo masculino. El tipo de discapacidad prevaleciente fue la visual ( $41 \%$ ), en menor medida la física $(32 \%)$, le continúa la auditiva $(18 \%)$ y, por último, quienes tienen otros tipos de discapacidad (intelectual y enfermedades discapacitantes) con un porcentaje muy bajo (9\%). El 60\% de la muestra presenta edades de entre 20 y 25 años.

$\mathrm{Al}$ momento del relevamiento, la mayoría de los estudiantes en ambas instituciones, se encontraba cursando el tercer y cuarto año de sus respectivas carreras y, según el año de ingreso declarado, puede afirmarse que los encuestados recorren un trayecto típico y acorde a lo esperado por la organización de los diversos planes de estudios.

En tanto a la situación académica, se describe a partir de promedios y, dado que se expresan en decimales, se consideró oportuna su división en rangos.

Esta primera aproximación permite advertir que la mayor concentración se encuentra en el rango de 7 a 8.99 con un 68\%, continuando los promedios comprendidos entre 5 y 6.99 con un $25 \%$ que, aunque son calificaciones más bajas, cuentan con la aprobación. En menor medida vemos aquellos casos aislados de un 5\% para los que tienen un promedio entre 9 y 10 y, por último, un 2\% para el rango de 3 a 4.99 . 
Tabla 3. Distribución de porcentajes según promedio académico

\begin{tabular}{|l|c|c|c|}
\hline & Total & UNcuyo & UNSa \\
\hline Promedio académico & $\%$ & $\%$ & $\%$ \\
\hline 0 a 2.99 & 0 & - & - \\
\hline 3 a 4.99 & 2 & 5 & 37 \\
\hline 5 a 6.99 & 68 & 14 & 63 \\
\hline 7 a 8.99 & 5 & 71 & - \\
\hline 9 a 10 & 100 & 100 & 100 \\
\hline Total & & 100 & 3 \\
\hline
\end{tabular}

Fuente: elaboración propia.

Así, se observa que más del 90\% de los estudiantes con discapacidad presenta un rendimiento académico que oscila entre calificaciones bajas pero positivas hasta las mayores calificaciones (9 y 10).

\section{Fortalezas y debilidades de la universidad para el acceso, permanencia y posterior egreso de los estudiantes}

El acceso al campo universitario es uno de los principales focos de atención en materia de discapacidad. Sin embargo, resulta necesario poder reconocer desde las voces de los propios estudiantes cuáles son los principales obstáculos con los que se enfrentan al momento de emprender sus recorridos académicos.

La mayoría de los estudiantes con discapacidad se encuentra en cursos avanzados, por lo que resultó interesante emplear metodología de tipo cualitativa que posibilite indagar sobre los factores que hayan podido incidir en el acceso y permanencia de los participantes en la universidad, ya sea favoreciendo u obstaculizando sus recorridos. En este sentido, se les solicitó a los encuestados que indicaran enunciados vinculados a aquellos factores que favorecieron el proceso y a los que dificultaron el mismo. Esto arrojó un total de 138 palabras para identificar los elementos positivos y 104 que identifican a los negativos. Las 242 palabras fueron agrupadas en siete categorías que las contemplan:

Se puede observar que, si bien se abordan los mismos factores en un caso como favorecedores y, en el otro como obstaculizadores, se encuentra una extensa distribución (gráfica 1). Agudizando un poco la mirada es notorio que en cuanto a acceso, es evidente la distancia entre los dos factores comparados, siendo su elevado porcentaje $(20.19 \%)$ el que lo define como un elemento que obstaculiza el ingreso y permanencia en la universidad. Esta categoría en su valoración como dificultad, refiere a los recursos de infraestructura, físicos, curriculares e informacionales insuficientes y/o inexistentes. 
En cuanto a la categoría Factor humano, los estudiantes rescataron un valor positivo por parte de éste en el acceso y permanencia en la universidad. Su porcentaje prácticamente duplica a la misma categoría en su identificación como limitación (16.67\% frente a $8.65 \%$ ). En este sentido, la categoría alude a la vinculación o contacto con otras personas, interacción que en algunos casos fue positiva - la mayoría- y en otros, negativa.

Los Factores actitudinales concentran el 25\% de las respuestas totales y tienen un porcentaje casi parejo para una y otra connotación $(26.81 \%$ como facilitadores y $24.04 \%$ como obstaculizadores). Esta categoría refiere a las actitudes de las personas del entorno para con los estudiantes con discapacidad. Tales actitudes tienden a fluctuar, así como las percepciones que de las mismas se puedan tener. Tanto la valoración de unos como de los otros depende de las percepciones individuales, por lo que no asombra su fluctuación entre una connotación y la otra.
$\mathrm{Al}$ igual que la categoría anterior, se presenta $R e^{-}$ cursos económicos con valores similares: $7.97 \%$ como aspecto favorecedor y $8.65 \%$ como dificultad.

Estos datos también dan cuenta de las particularidades y heterogeneidad de la población con la que se trabajó, puesto que lo que resulta facilitador para algunos, no lo es para otros.

Tanto la categoría Aspectos legales como Aspectos organizacionales se manifiestan más como obstáculos. En el caso de la primera, los sujetos destacaron la falta de actualización y aun ausencia de legislación, norma o política de contención hacia los estudiantes en general y, especialmente, de los que tienen discapacidad. Para la segunda categoría, los encuestados indicaron la escasa o mala distribución de tiempos y espacios dentro del ámbito académico (aulas pequeñas con exceso de alumnos, sobrecarga horaria de materias, regímenes de correlatividades, entre otros), lo cual influye negativamente en sus trayectos formativos.

\section{Gráfica 1. Porcentaje según factores facilitadores y obstaculizadores del acceso y permanencia en la universidad}

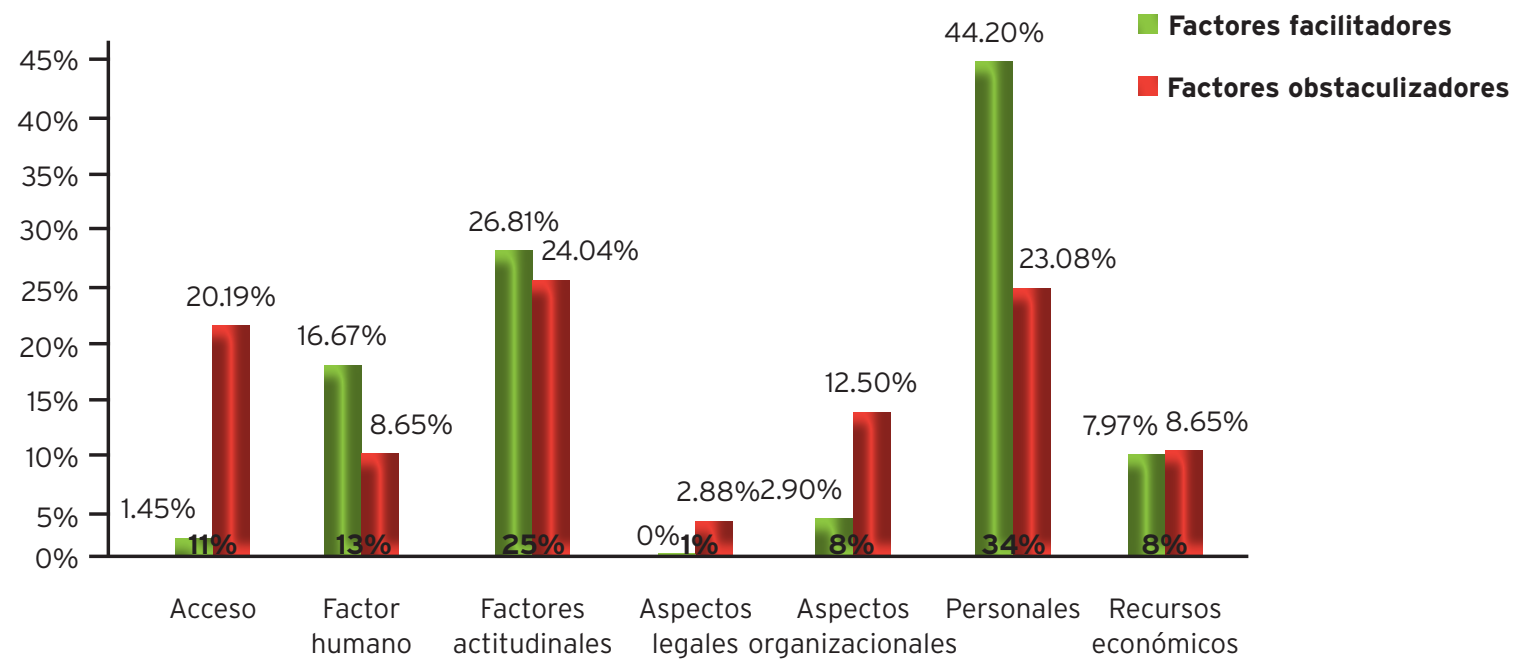

Fuente: elaboración propia. 
Por último, puede observarse en el gráfico que se destaca la barra de Factores personales como elemento positivo que favorece el acceso y permanencia en la educación superior. Tales factores están ligados a cuestiones exclusivamente del sujeto, referidas al autoconcepto, a las emociones y sensaciones experimentadas por el propio estudiante. La categoría aludida concentra entre sus dos distribuciones un $34 \%$ del total de respuestas, lo que indica que la mayoría de los estudiantes con discapacidad, se reconocen como los propios responsables, para bien o para mal, de facilitar o dificultar el acceso y permanencia a la educación superior. Aquí se encuentran algunas palabras que describen su incidencia como aspectos positivos en el trayecto, tales como: esfuerzo, interés, constancia, mérito, lucha, motivación, perseverancia (es la más destacada) y voluntad, entre otras. Por otro lado, contrariamente, se declaran vocablos con incidencia negativa, tales como: baja autoestima, indecisión, "dejadez", fobia, enfermedades, desmotivación, desconcentración, aislamiento, trabas personales, silencio - no dar a conocer la discapacidad-, cansancio y cuestiones de salud como las más representativas. Puede advertirse, de acuerdo con la última descripción, que muchos estudiantes aludieron directa o indirectamente a su discapacidad o momentos asociados a ésta, como los principales factores que obstaculizaron su acceso y permanencia en la universidad.

\section{Autoestima}

Desde el punto de vista cuantitativo partimos de los siguientes índices descriptivos por dimensión de la variable: media, desviación estándar, mediana, puntaje mínimo y puntaje máximo; luego se grafican los valores medios y la distribución de las puntuaciones a través de un diagrama de caja. Finalmente, se interpreta la puntuación total en autoestima a partir de la categorización del autor del instrumento (ToroVargas, 1994).

Dado el número disímil de ítems entre las dimensiones del instrumento, se convierten a escala 10 para facilitar el análisis y la comparación (véanse tabla 4 y gráfica 2).

Tabla 4. Valores descriptivos de las seis escalas

\begin{tabular}{|l|c|c|c|c|c|c|}
\hline \multicolumn{1}{|c|}{ Escala } & Casos & Media & D.E. & Mediana & $\begin{array}{c}\text { Puntaje } \\
\text { mínimo }\end{array}$ & $\begin{array}{c}\text { Puntaje } \\
\text { máximo }\end{array}$ \\
\hline Dimensión afectiva (+) & 40 & 6.53 & 1.25 & 6.67 & 4.17 \\
\hline Dimensión socio-afectiva (-) & 40 & 4.96 & 1.23 & 4.75 & 3 & 8.75 \\
\hline Dimensión ética (-) & 40 & 3.99 & 1.11 & 3.75 & 2.50 & 6.88 \\
\hline Dimensión ética (+) & 40 & 8.00 & 1.28 & 8.13 & 5 & 10 \\
\hline Dimensión cognoscitiva (+) & 40 & 8.10 & 1.31 & 8.25 & 5 & 10 \\
\hline Dimensión física (-) & 40 & 4.23 & 1.55 & 4.38 & 2.50 & 10 \\
\hline
\end{tabular}

Fuente: elaboración propia, a partir del análisis efectuado con el SPSS. 


\section{Gráfica 2. Valores medios de las seis escalas}

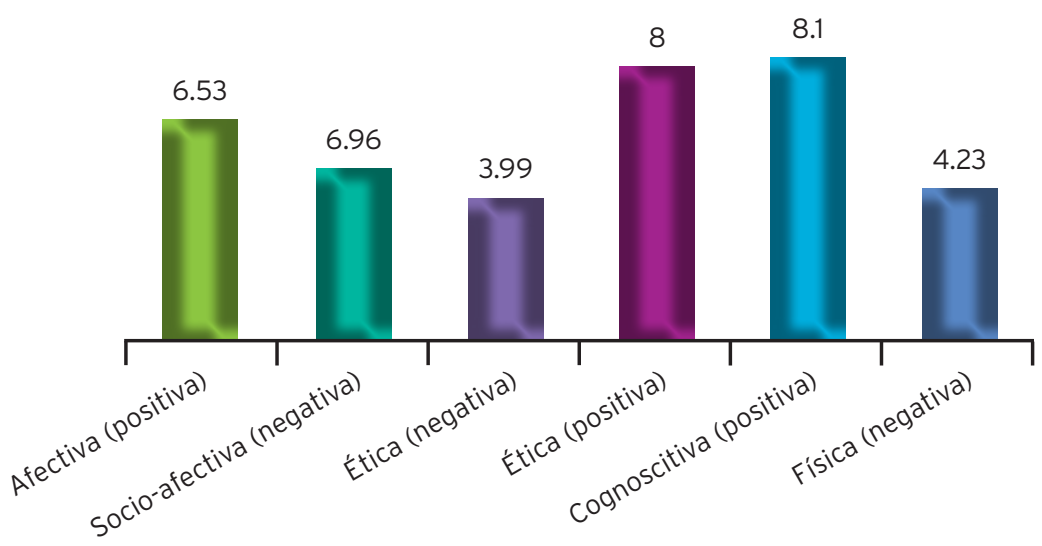

De las seis dimensiones de autoestima, gratamente la que muestra la media más baja es la dimensión ética negativa (soberbia) y las que evidencian las más altas son también la ética pero positiva y la escala cognoscitiva. La otra dimensión de signo positivo manifiesta un valor medio módico.

Finalmente, se representa la distribución de las puntuaciones por escala (gráfica 3). Se advierte que las dimensiones positivas se corren claramente a la derecha, siendo la afectiva la que evidencia un grado menor de autoestima en comparación con las otras dos. En cambio, las negativas se ubican a la izquierda (hacia puntuaciones más bajas). De importancia para tareas de orientación educacional son dos estudiantes con dificultades marcadas en el área socioafectiva y física de la autoestima, respectivamente.

\section{Gráfica 3. Diagrama de caja de las seis escalas}

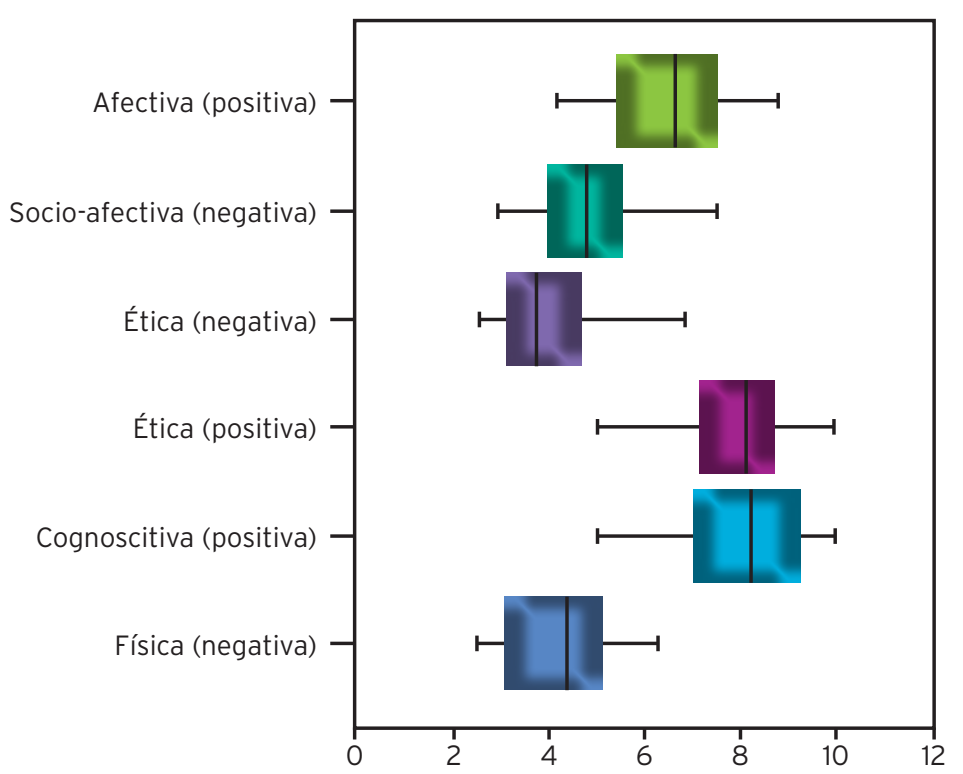




\section{Interpretación del autoexamen}

Según se señaló, la sumatoria de las puntuaciones de la totalidad del instrumento (40 ítems) se agrupan en cuatro categorías de autoestima. Desde esta categorización del autor (Toro-Vargas, 1994), los resultados pueden verse en la tabla 5 .
Es evidente que predomina la autoestima alta positiva que, junto con la baja positiva, representan al 90\% de los alumnos. Son casos excepcionales los que hacen al 10\% restante.

Tabla 5. Categorías de autoestima

\begin{tabular}{|l|c|c|}
\hline \multicolumn{1}{|c|}{ Autoestima } & Frecuencia & Porcentaje \\
\hline Baja negativa & 3 & 7.5 \\
\hline Baja positiva & 13 & 32.5 \\
\hline Alta positiva & 23 & 57.5 \\
\hline Alta negativa (sobreestima) & 1 & 2.5 \\
\hline Total & 40 & 100.0 \\
\hline
\end{tabular}

Fuente: elaboración propia.

\section{Gráfica 4. Categorías de autoestima en porcentaje}

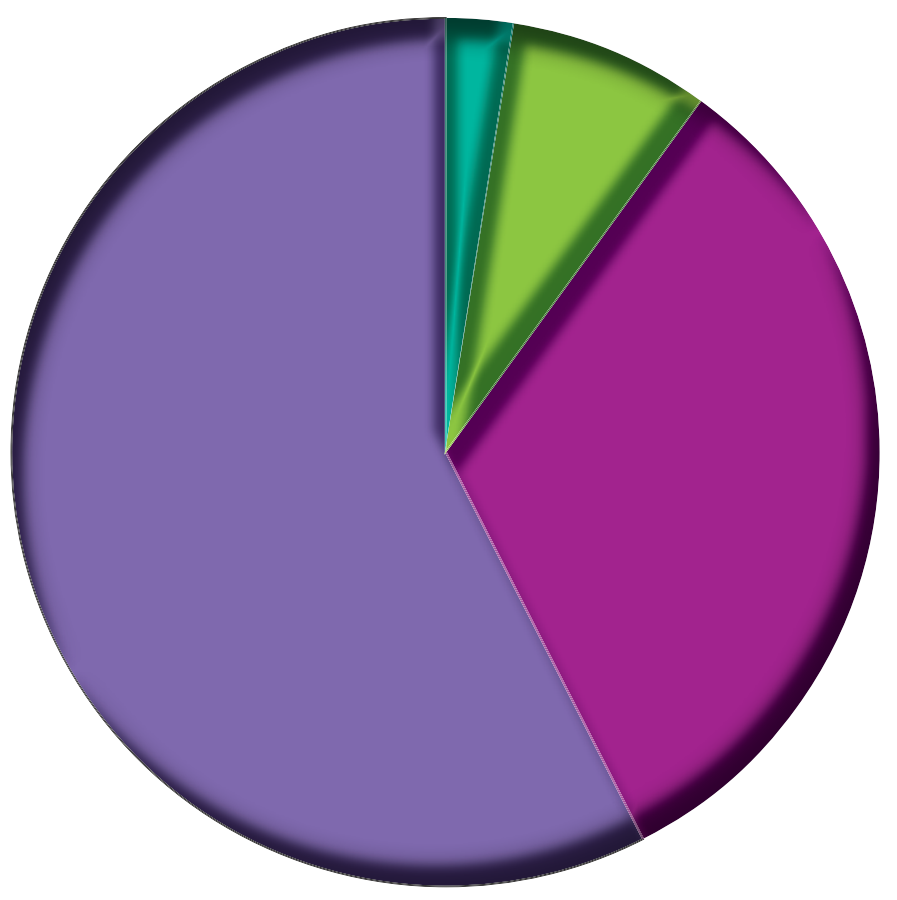




\section{Relación entre autoestima $y$ rendimiento académico}

A fin de ponderar las asociaciones entre las variables autoestima y desempeño académico, se efectuó un análisis en tres conglomerados de $k$ medias limitado a la operación "clasificar" por el tamaño de la muestra. Resulta importante destacar en este apartado que tal análisis se realizó abordando la muestra completa sin distinción por tipo de discapacidad puesto que su contemplación no arrojó resultados significativos (tabla 6).

Recorriendo los valores alcanzados, los dos conglomerados de mayor distribución porcentual son el 2 y 3 . No obstante, el 1 presenta un valor del 15\% del total, referenciando a aquellos estudiantes con discapacidad que parecen estar definidos por aspectos, en su mayoría, negativos.

En la tabla 7 se presenta la conformación de los conglomerados finales integrando las variables bajo estudio (dimensiones de autoestima y rendimiento).

Tabla 6. Distribución de los conglomerados

\begin{tabular}{|l|c|c|c|}
\cline { 3 - 4 } \multicolumn{2}{c|}{} & $N$ & $\%$ del total \\
\hline Conglomerado & 10 & 6 & $15 \%$ \\
\hline & 20 & 16 & $40 \%$ \\
\hline Total & 30 & 18 & $45 \%$ \\
\hline
\end{tabular}

Fuente: elaboración propia, a partir del análisis efectuado con el SPSS.

Tabla 7. Centros de los conglomerados finales

\begin{tabular}{|l|c|c|c|}
\cline { 2 - 4 } \multicolumn{1}{c|}{} & \multicolumn{3}{c|}{ Conglomerado } \\
\hline \multicolumn{1}{c|}{ Variables } & $\mathbf{1}$ & $\mathbf{2}$ & $\mathbf{3}$ \\
\hline Afectiva + & 6.81 & 6.78 & 6.30 \\
\hline Socio-afectiva - & 7.92 & 4.63 & 4.72 \\
\hline Ética - & 3.96 & 3.87 & 4.05 \\
\hline Ética + & 6.88 & 8.07 & 8.13 \\
\hline Cognoscitiva + & 8.17 & 8.22 & 7.97 \\
\hline Física - & 6.04 & 4.03 & 4.17 \\
\hline Rendimiento & 5.81 & 7.68 & 7.33 \\
\hline
\end{tabular}

Fuente: elaboración propia, a partir del análisis efectuado con el SPSS. 
De acuerdo con las características obtenidas de la población investigada y, según lo analizado, se advierte la constitución de dos perfiles psicosociales claramente demarcados. Por un lado y, en correspondencia con el primer conglomerado, se halla un primer perfil caracterizado por aspectos negativos: dimensión socio-afectiva negativa alta, dimensión ética positiva más baja que los otros dos conglomerados, física negativa más alta y evidencia el rendimiento inferior de la muestra. Por otro lado, los valores arrojados por el segundo y tercer conglomerado posibilitan su unificación conformando un perfil representado por aspectos positivos: puntuación baja tanto en el área socio-afectiva negativa como en la física negativa de la autoestima, una dimensión ética positiva alta y un rendimiento superior, siendo ligeramente más alto el del segundo conglomerado.

\section{Discusiones y conclusiones}

La postura sostenida a lo largo de este trabajo se enmarca desde un modelo social que explica que, cualquier persona con alguna deficiencia tendrá una discapacidad si encuentra alguna dificultad o imposibilidad para realizar una o más actividades de la vida cotidiana, debido a las barreras que la sociedad le impone (Palacios, 2008; Soto-Builes et al., 2008, González-Lizárraga, 2011). Bajo este enfoque, la cuestión de la accesibilidad (en todas sus dimensiones) tiene una impronta fundamental.

En este sentido, a través de lo analizado, la mayoría de los estudiantes universitarios con discapacidad argumenta que no sólo las condiciones edilicias o de infraestructura de los campos académicos no son óptimas para recibir a personas con distintos tipos de discapacidad, sino también la ausencia o incumplimiento de normativas específicas y los comportamientos y actitudes del entorno, imponen las principales barreras para su acceso y permanencia en las instituciones. De acuerdo con los hallazgos de otros estudios, se destaca que entre estas barreras se encuentran, además de los obstáculos para la movilidad, los obstáculos urbanísticos y de transporte, las actitudes sociales estereotipadas, la falta de oportunidades y la dificultad de acceso a los recursos (Botero-Soto et al., 2013). En esta línea, se manifiesta que mucho de lo actitudinal puede relacionarse con el gran desconocimiento que hay sobre la temática de la discapacidad y, por ende, el temor que surge en algunos individuos al tener que abordarla (Díaz et al., 2004; Díaz-Jiménez, 2004).

No obstante, deben realizarse dos salvedades al respecto: la primera, al considerar que algunos encuestados también se adjudicaron la responsabilidad, ya sea como obstáculo o como fortaleza, en el acceso y permanencia en las universidades. Es decir, enfatizaron que son ellos quienes, por distintos factores personales, actúan como barreras o como facilitadores de los procesos. Muchos de aquellos que se responsabilizaron como obstáculo en su trayecto, lo ligaron a cuestiones asociadas con la propia discapacidad. En segundo lugar, cabe resaltar que, pese a que las condiciones no están del todo dadas para incluir plenamente a las personas con discapacidad, los esfuerzos de las instituciones o de algunas personas dentro de ellas por conseguir esto, se están vislumbrando paulatinamente lo cual, a su vez, es también reconocido por los estudiantes.

En continuidad con lo expresado, algunos autores coinciden en que la gestación y constitución de una cultura inclusiva supone un cambio significativo y profundo, convirtiéndose en una tarea difícil que nos involucra a todos. Actualmente se pretende que las instituciones educativas sean capaces de lograr la adaptación de la oferta formativa a la diversidad del estudiantado (Echeita, 2007; Valcarce, 2010).

En este sentido, la organización institucional mediante los diferentes servicios que ofrece, aporta en gran medida para intentar suprimir las barreras que limitan la realización de un trayecto formativo en igualdad de condiciones respecto a las personas sin discapacidad. Así pues, encontramos diversas propuestas que realiza cada universidad para la 
totalidad de su estudiantado en general, y para los que tienen discapacidad, en particular. La Universidad Nacional de Cuyo presenta gran avance en cuanto a normativas, programas específicos (cuenta con actividades de extensión dirigidas a la adecuación de materiales, a la formación de personas en el área, becas pre-profesionales para insertar a los estudiantes en el ámbito laboral, etcétera) y servicios especiales para personas con discapacidad. En la Universidad Nacional de Salta, los pasos son más incipientes aunque de gran significancia: la conformación de la Comisión de Inclusión de Personas con Discapacidad permitió que comenzaran a debatirse y llevar a cabo algunas modificaciones edilicias, políticas, culturales y pedagógicas que promueven la contención de las personas con discapacidad, lo cual alienta la neófita visibilidad que están adquiriendo estos estudiantes en la institución.

De lo hasta aquí dicho se desprende, como se sostiene en algunos trabajos - que no es casual que sean escasos-, que los sujetos con discapacidad que acceden a la educación superior consideran que las condiciones propicias para una verdadera inclusión están en desarrollo. Al mismo tiempo, son los propios estudiantes los que empezaron a hacerse presentes, demostrando sus capacidades, posibilidades, autonomía y haciendo valer sus derechos (Celada, 2006; Palacios, 2008, Echeita 2007).

\section{Perfil de los estudiantes con discapacidad en relación a la autoestima}

En cuanto a autoestima, es necesario recapitular que ésta ha sido evaluada en función de seis dimensiones - tres positivas y tres negativas - y, también, según la clasificación que brinda Toro-Vargas (1994). A partir de esta última distinción, casi la totalidad de los estudiantes con discapacidad tienen una autoestima positiva. Casos excepcionales son los que se destacan por tener una autoestima negativa - en un caso sobreestima en otros falta de estima.
Que la mayoría de los sujetos tengan valores positivos, quiere decir que cuentan con una personalidad más plena y una percepción más satisfactoria de la vida. Una autoestima positiva aumenta la capacidad de afrontar y superar las dificultades personales, fomenta la capacidad de adquirir compromisos y por lo tanto de ser más responsables. Cuando el límite de la autoestima es negativo pero con una valoración positiva, refiere a que la persona presenta ciertos grados de inseguridad sobre sí misma, pero eso no le impide fijarse metas y aspiraciones propias, superar dificultades y tener una percepción medianamente satisfactoria de la vida (Toro-Vargas, 1994).

Esa pequeña porción de estudiantes con una autoestima negativa, implica que presentan escaza confianza en sí mismos para sortear, desde una perspectiva positiva, los obstáculos, problemas y conflictos que pudieran presentárseles y esto ocasiona que la calidad de vida no sea tan óptima como pudiera serlo. La falta de confianza genera la evitación o evasión de compromisos y, por lo tanto, o se abordan nuevas responsabilidades, privándose de otras experiencias vitales, o bien, cuando se asumen nuevos compromisos abruman las responsabilidades siendo el umbral de resistencia al conflicto más limitado. No contribuye a fomentar la creatividad, puesto que no se confia en las propias capacidades, por lo que se es más propenso a actuar de acuerdo con lo que se espera de uno y no de acuerdo con las propias decisiones (Toro-Vargas, 1994). En el polo opuesto se encuentra aquel sujeto que resultó manifestar un exceso de autoestima, lo cual se traduce en actitudes individualistas, suele percibirse por encima de los demás y tiende a la subestimación y/o indiferencia para con los otros y el entorno.

Salvo por estos sujetos que presentan una connotación negativa, se puede decir que los resultados alcanzados encuentran cierta disonancia con otros trabajos en los que se obtiene que los estudiantes con discapacidad tienen una percepción más negativa de su estado emocional y estima, tanto en general como en situaciones específicas (Polo et al., 2012). 
En consideración de las dimensiones de la autoestima, se advierte que la ética positiva $(+)$ y cognoscitiva, evidencia los grados más elevados de autoestima, mientras que la afectiva presenta puntuaciones más bajas que estas dos. La dimensión ética negativa (-), socio-afectiva (-) y física (-), manifiestan valores más bajos de autoestima. Esto quiere decir que, de las seis dimensiones, la que presenta puntajes más bajos es la ética negativa, señalando que son casos extraordinarios los que tienen conductas soberbias, en tanto, las dimensiones positivas indican que los estudiantes demuestran cierta seguridad acerca de lo que se considera bueno y de las conductas correctas que se deben realizar para las personas y para la sociedad, y aceptan responsabilidades, errores y aciertos; tienen confianza en sus capacidades y se muestran receptivos a adquirir nuevos conocimientos, nuevas experiencias y, por último, son capaces de expresar los sentimientos, sensaciones, temores y de establecer vínculos afectivos reconocidos como valiosos entre compañeros, amigos y familiares, aunque con menos frecuencia. En cuanto a la valoración del aspecto físico, la autoestima tiende a ser relativamente baja. Caso contrario a lo hallado en otra investigación realizada con personas con discapacidad física específicamente, en donde se obtiene que éstas presentan una valoración positiva de su imagen corporal, especialmente en la esfera de la satisfacción con el cuerpo (Botero-Soto et al., 2013).

\section{Perfil de los estudiantes con discapacidad en relación con la autoestima y el rendimiento académico}

En relación con estas dos variables encontramos que los estudiantes con discapacidad agrupados bajo el segundo perfil, tienden a un rendimiento académico (en función de sus promedios) más alto que aquellos que conforman el primer perfil, caracterizado en su generalidad por aspectos negativos. Sin embargo, cabe aclarar respecto a este dato que, en ambos grupos, la dimensión cognoscitiva de la autoestima es elevada y por ende el desempeño académico oscila de regular a bueno (5-6) para el primero y de bueno a muy bueno (7-8) para el segundo.

De esto se desprende que el perfil de los sujetos del grupo uno, aunque presentan una confianza relativamente positiva en sus capacidades, el resto de sus características tienden a ser negativas, por lo que su autoconcepto académico resulta más bajo. En tanto, el perfil de los estudiantes con discapacidad del grupo dos, se caracteriza por presentar lo que se denomina un autoconcepto académico positivo, lo cual se acompaña de una autoestima positiva, en la confianza depositada en sus capacidades (dimensión cognoscitiva), así como en la generación de expectativas más ambiciosas y en las motivaciones que responden a intereses personales. Al respecto coincidimos con lo hallado en la investigación de Polo et al. (2012) con relación a que la valoración que la persona sostiene sobre su capacidad, su autoestima y autoeficacia incide en su motivación hacia los aprendizajes y moldean su éxito en este ámbito. En este sentido, se evidencia que factores personales tales como el autoconcepto y la autoestima tienen cierto impacto en las trayectorias académicas de los educandos, dada sus vinculaciones con las percepciones, expectativas y motivaciones de los mismos. Es posible que la consideración de este constructo pueda colaborar en la previsión no sólo del rendimiento de los estudiantes, sino también de su vulnerabilidad física y psicológica (Polo et al., 2012). Así, la función de la educación se torna esencial para la generación y promoción de estrategias encaminadas a fortalecer las potencialidades de cada sujeto y erradicar las limitaciones que impiden el desarrollo de una calidad de vida plena.

El presente estudio puso de relieve y dio lugar a la discusión sobre ciertos aspectos que pueden intervenir en la configuración de los perfiles psicosociales de los estudiantes universitarios con discapacidad, reflexionando sobre la influencia que los factores 
contextuales, psicosociales y personales pueden tener en las decisiones, motivaciones, elecciones, expectativas, así como en la conformación de sus recorridos formativos y de sus desempeños personales y profesionales.

Finalmente, se remarca que los resultados expuestos en este trabajo y las discusiones suscitadas no pretenden ser generalizaciones de los perfiles destacados de los estudiantes con discapacidad y sus recorridos universitarios, puesto que esto no se correspondería con la consideración de la diversidad

\section{Referencias}

Abric, Jean Claude (2001), Prácticas sociales y representaciones, México, Ed. Coyoacán.

Aparicio, Miriam (2009), La demora en los estudios universitarios. Causas desde una perspectiva cuantitativa, Mendoza, UNCuyo- EDIUNC.

Baranger, Denis (2009), Construcción y análisis de datos. Introducción al uso de técnicas cuantitativas en la investigación social, Posadas, Editora Universitaria de Misiones.

Botero-Soto, Paula y Londoño-Pérez, Constanza (2013), "Factores psicosociales predictores de la calidad de vida en personas en situación de discapacidad física", en Revista Acta colombiana de psicología, 16 (2), Colombia, Universidad Católica de Colombia, pp. 125-137.

Castilla, Mónica (2006), "Competencias del docente de educación especial ante las nuevas concepciones de la relación educación-trabajo", en Revista Colección Ensayos y Experiencias. Educación Especial: Inclusión educativa. Nuevas formas de exclusión, Buenos Aires, Novedades Educativas, pp. 85-95.

Castro-Solano, Alejandro y María Martina Casullo (2001), "Rasgos de personalidad, bienestar psicológico y rendimiento académico en adolescentes argentinos", en Interdisciplinaria, 18 (1), Argentina, Centro y la revalorización de las diferencias a la que adherimos. Por el contrario, lo que se subraya en esta investigación es que, tanto los perfiles psicosociales como las trayectorias académicas, dependen de un contexto, se configuran y desarrollan de acuerdo con la particularidad de espacios, tiempos, personas, aspectos y momentos. En este sentido, cobran especial relevancia en el presente estudio las trayectorias y perfiles psicosociales de estudiantes con discapacidad en dos contextos específicos: la Universidad Nacional de Salta y la Universidad Nacional de Cuyo.
Interamericano de Investigaciones Psicológicas y Ciencias Afines, pp. 65-85, <http://www.redalyc.org/ articulo.oa?id=18011326003> [Consulta: agosto de 2014].

Celada, Beatriz (2006), "Educación superior y alumnos universitarios con discapacidad", en Revista Colección Ensayos y Experiencias. Educación Especial: Inclusión educativa. Nuevas formas de exclusión, Buenos Aires, Novedades Educativas, pp. 71-84.

Díaz-Jimenez, Gabriel (2004), Los estudiantes con discapacidad en la Universidad de Las Palmas de Gran Canaria, España, Universidad de las Palmas de Gran Canarias- Vicerrectorado de Planificación y Calidad.

Díaz, Liliana y Claudia López (2004), "Estudio sobre las concepciones y acciones relativas a la discapacidad en la Universidad Nacional de Mar del Plata", en Dell'Anno, Corbacho y Serrat (comp.), Alternativas de la diversidad social: las personas con discapacidad, Argentina, Espacio Editorial, pp. 175-217.

Echeita-Sarrionandia, Gerardo (2007), Educación para la inclusión o educación sin exclusiones, Madrid, Nercea Ediciones.

Garrido, Álvaro (2012), Significados de una trayectoria 
educativa. Evaluación del "éxito" y el "fracaso" educativo desde la reconstrucción subjetiva de adultos de la $V$ Región, Chile, Universidad de Valparaiso, <http://www.slideshare.net/alvaroleongarrido/ significados-de-una-trayectoria-educativa? utm_ source=slideshow02\&utm_medium $=$ ssemail\&utm_ campaign=share_slideshow_loggedout\#> [Consulta: diciembre de 2014].

González-Lizárraga, María Guadalupe (2011), Les trajectoires des étudiants universitaires: un modèle intégré [Las trayectorias de los estudiantes universitarios: un modelo integral], Montreal, Université de Montréal, Département d'administration et fondements de l'éducation, Faculté des sciences de l'éducation.

Hernández-Sampieri, Roberto, Carlos FernándezCollado y Pilar Baptista-Lucio (2010), Metodología de la investigación (5ta. ed.), México, McGraw-Hill.

Huanco-Flores, Cármen (2004), "Nivel de autoestima de los pacientes del programa de control de tuberculosis del Centro de Salud Laura Rodríguez Dulanto-Duksil", Tesis de Licenciatura, Lima- Perú, Universidad Nacional Mayor de San Marcos.

Kaplan, Carina y Paula Fainsod (2001), "Pobreza urbana, diversidad cultural y escuela media. Notas sobre trayectorias escolares de las adolescentes embarazadas", en Revista del Instituto de Investigaciones en Ciencias de la Educación IICE, 10 (18), Argentina, Mimeo, pp. 25-36.
Orell, José y Daniel Sainz (2006), "Riesgos psicosociales en la Universidad", en Seminario permanente del Grupo de Trabajo sobre Calidad Ambiental y Desarrollo Sostenible sobre Riesgos Psicosociales, Málaga, Universidad de Málaga.

Palacios, Agustina (2008), El modelo social de discapacidad: orígenes, caracterización y plasmación en la Convención Internacional sobre los Derechos de las Personas con Discapacidad, España, CERMI, Ed CINA.

Polo, María Tamara y María Dolores López (2012), "Autoconcepto de estudiantes universitarios con discapacidad visual, auditiva y motora", en Revista Latinoamericana de Psicología, 44 (2), España, pp. 87-98.

Serrano-Barquín, Carolina, Adelaida Rojas-García y Camilo Ruggero (2013), "Depresión, ansiedad y rendimiento académico en estudiantes universitarios", en Revista Intercontinental de Psicología y Educación, 15 (1), Universidad Intercontinental, México, pp. 47-60, <http://www.redalyc.org/articulo. oa?id=80225697004> [Consulta: julio de 2013].

Soto-Builes, Norelly y Carlos Vasco (2008), "Representaciones sociales y discapacidad", en Revista Hologramática, año V, 1 (8), Colombia, Universidad de Manizales- Facultad de Ciencias Sociales, pp. 3-23.

Toro-Vargas, Cirilo (1994), Nuevos Surcos, Ponce, Puerto Rico, Ediciones Guayacán.

Valcarce, Margarita (2011), "De la escuela integradora a la escuela inclusiva", en Revista Innovación Educativa, núm. 21, pp. 119-131.

Cómo citar este artículo:

Mainardi-Remis, Ana-Inés (2018), "Incidencia de factores institucionales y de la autoestima en las trayectorias académicas de estudiantes con discapacidad", en Revista Iberoamericana de Educación Superior (RIES), México, UNAM-IISUE/Universia, vol. IX, Núm. 26, pp. 171-190, [consulta: fecha de última consulta]. 